\title{
STRUCTURE AND NATURE OF AGNS
}

\author{
Hagai Netzer \\ School of Physics and Astronomy and The Wise Observatory \\ Tel Aviv University, Tel Aviv 69978, Israel.
}

\begin{abstract}
This review discusses the present understanding of the inner region of AGN. Several fundamental questions are adressed, such as the determination of the central mass, the accretion rate and the presence or absence of massive accretion disks. The "standard model", used until recently to deduce the density, ionization, geometry and other physical properties of AGN, is facing sever difficulties and several unsolved problems, such as the hydrogen line ratio, the FeII spectrum, the energy budget discrepency and the confinement of the broad line clouds may force us to abandon it all together. The new line-continuum reverberation measurements are perhaps the most important developement of recent years and their significance is discussed in relation to the other mentioned problems. Thin or thick accretion disks are likely to be present in the very center of AGNs. The theory of such disks is not advanced enough to provide accurate spectral calculations and the best way to identify them is to look for the signature of their nonisotropic radiation field in well selected samples. Several new developements and ideas, such as disk shaped emission line region, stars and stellar winds as broad line clouds, dust emission and very small-very dense BLR are also discussed.
\end{abstract}




\section{Fundamental Questions}

In studying Active Galactic Nuclei (AGN) we are concerned with several fundamental questions about their structure, evolution and the nature of their central source. Since a general, comprehensive theory is not yet available we adopt an empirical approach, trying to answer simpler questions, and secure basic data. Thus the basic question of "what is the nature of the central source?" is replaced by "what is M?" (its mass), for which we may find an answer.

This review is concerned with the innermost part of AGN, up to the outer limit of the Broad Line Region (BLR). Besides the central massive object and the BLR clouds, this region may or may not contain an accretion disk and hot, dilute gas (the "hot phase"). The fundamental issues I shall address, and the simpler, more specific related questions, (in parentheses) are:

1. What is the nature of the central source? (what is M?)

2. What is the accretion mechanism? (what is the accretion rate, $\dot{m}$ ? ).

3. How do clouds formed and destroyed? (Is there a "hot phase"?)

4. Are there massive accretion disks in AGN? (What is the origin of the UV continuum? Do we see inclination effects?).

5. What is the origin and nature of relativistic motions? (Have beaming phenomena or jets been detected?).

Much of the information required to answer these and other questions comes from spectral analysis. Detailed methods for analyzing the data have been developed and a coherent picture, sometimes referred to as "The Standard Model", emerged. In this short review there is no room to address all important topics and some, such as the issue of cloud motion and dynamics, will be omitted. Also, there is no way to mention all those contributed to the developement of the model and I shall note mainly new references, since 1985. Comprehensive reviews, by Davidson and Netzer 1979, Ferland and Shields, Mathews and Capriotti and Osterbrock and Mathews (1986) provide full account of the situation prior to 1985 .

\section{The Standard Model}

The standard model provides a comprehansive description of the geometry, structure and physical processes in the center of AGNs. Its basic ingredients go back to the work of Davidson (1972) and MacAlpine (1972), but there have been many improvements since. The observational data base includes some 30 well-observed emission lines and the continuum energy distribution over the wavelength range $0.1-3 \mu$. Most line ratios do not differ much from object to object so the phrases "typical object" or "typical spectrum" are meaningful and a mean spectrum will be discussed. One such "typical spectrum is shown in Fig. 1.

Some line ratios, such as CIII 977/CIII] 1909 , indicate kinetic temperatures of the order of $10,000 \mathrm{~K}$, typical of photoionized gas. The ionizing continuum, although not directly observed, is definitely non-stellar in origin, and most calculations assume it to be a power-law in energy, or a combination of several such components.

Several other conclusions immediately follow: Typical densities are $10^{8}-10^{10} \mathrm{~cm}^{-3}$, 
as deduced from the relative strength of permitted and intercombination lines. Most lines have similar profiles, thus small, optically thick clouds are thought to produce all high and low excitation lines. The strong low excitation lines indicates large column densities, of the order of $10^{22-23} \mathrm{~cm}^{-2}$. X-ray heating and ionization must play an important role. Standard BLR clouds are assumed to have a highly ionized part as well as a warm, partly neutral zone, where most of the low excitation lines are formed. The large column density, high density and strong radiation field imply large optical depth in many resonance lines, as well as in the hydrogen Balmer, Paschen and higher series lines. Clouds must be confined, so a hot $\left(\sim 10^{8} \mathrm{~K}\right)$ confining medium is an additional important component.

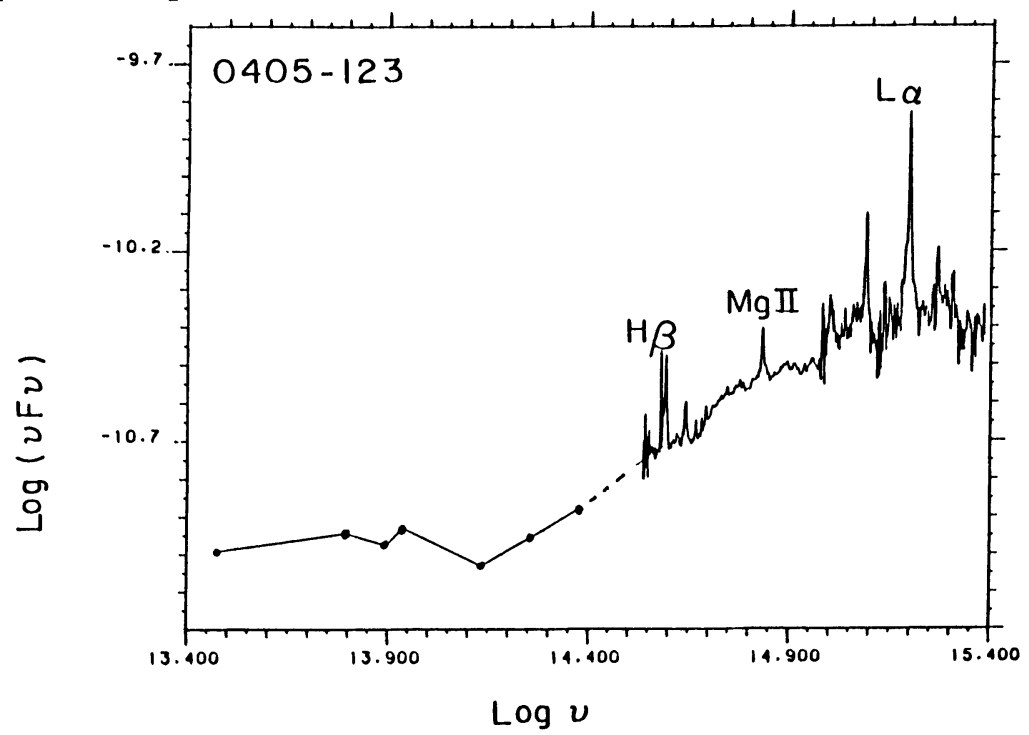

The IR-optical-UV spectrum of a typical quasar. Note the "blue bump" between 0.1 and $1 \mu$, the "small bump" which is the energy excess around MgII 2798 and the $3 \mu$ bump

All this sets the stage for photoionization model calculations. Such models are designed to mimic the physical processes and reproduce the observed spectrum. There re two stages to the calculation. First a model for one cloud is computed, defined by:

a. The ionization parameter, defined here as:

$$
U=\frac{\text { ionizing photon flux }}{N c}=\frac{Q}{4 \pi r^{2} N c}
$$

where $N$ is the hydrogen number density, $c$ the speed of light, $Q$ the total number of ionizing photons and $r$ the distance to the center.

b. The continuum energy distribution at all energies.

c. The column density of an individual cloud.

d. The chemical composition.

The result is the calculated line spectrum. In the second stage, a cloud distribution is assumed and the contribution of many individual clouds added. At this stage it is 
usefull to specify the covering factor, which is the solid angle sustended by optically thick clouds. Denote $C(r)$ the cumulative covering factor up to distance $r$, we can write:

$$
C(r)=r^{x}
$$

where $x$ depends on the density distribution and cloud velocity. For example, in a system of spherical clouds, moving in or out at their virial velocity, where the pressure is given by $p \propto r^{-\bullet}$, we find $x \simeq 2 / 3 s-1 / 2$. The best estimates for the total covering factor is about 10 percent, but the issue of the inner and outer bounderies of the BLR is not simple. Some natural pressure laws predict $s \simeq 2$ so $C(r)$ is peaked at the outer boundary of the BLR. Under these circumstances it is not a bad approximation to consider a single cloud, with the full covering factor, as representing the conditions across the BLR. by:

The single, or "mean" cloud picture results in a "mean radius" for the BLR, given

$$
r=\left[\frac{Q}{4 \pi N U c}\right]^{1 / 2} \simeq 3.5 \times 10^{17}\left[N_{10} U<\nu>\right]^{-1 / 2} L_{46}^{1 / 2}(\text { ion }) \mathrm{cm}
$$

where $N_{10}=N / 10^{10} \mathrm{~cm}^{-3}, L_{46}(i o n)$ is the ionizing luminosity in units of $10^{46} \mathrm{erg} / \mathrm{sec}$ and $\langle\nu\rangle$ is the mean energy (Ryd) of an ionizing photon.

The assumption of Keplerian motion gives an estimate of the central mass, since the velocity can be measured from the observed line profiles. All these estimates depend on the values of $U$ and $N$ that are obtained from the model.

The physics that goes into a model is rather complex. Hundreds of atomic processes must be included, some with no reliable cross sections. The statistical equilibrium equations for all ions, and the ionization structure of all elements must be solved and the line and continuum transfer calculated. The complex nature of this problem lead to some simplified assumptions that cannot always be justified. Several processes known to be important at high densities were not included until now, at least not for all elements, and a simplified transfer method, based on the escape probability formalism, is usually applied. Two parallel approaches have been developed. The first emphasizes the full treatment of all atomic processes, at the expense of the transfer calculations (e.g. Kwan and Krolik 1981, Mushotzky and Ferland 1984), the second uses an improved transfer method but many atomic processes are treated in an approximate way (e.g. Hubbard and Puetter 1985, Averett and Loeser 1988).

Considering all limitations, the success of the standard model has been quite remarkable. It explains many of the ultraviolet lines as well as the optical hydrogen and helium spectrum. The overall configuration, relating clouds dimension, location and velocity with the observed continuum and the deduced mass seemed to be in good agreement with the observations. Some difficulties has been noted from the very early days, especially the disagreement of the observed and calculated intensity of the high excitation lines, such as NV 1240 and OVI 1035. Recent developements brought about many other problems, as discussed in section 3.

The potential accuracy of a good photoionization model is so great that many of the results must be taken very seriously. A discrepency by 30 or 40 percent in certain line ratios is sometimes enough to rule out a model. Obviously some line intensities are not as accurate and a factor of two uncertainty is not uncommon. This must be remembered when we come to discuss the alternatives. 


\section{Outstanding Problems}

\subsection{The hydrogen line spectrum}

This problem, sometimes referred to as "the $L \alpha / H \beta$ problem", was noted by Baldwin (1977) and others later on and is still not solved. The standard model in its simplest form predics $\mathrm{I}(L \alpha) / \mathrm{I}(H \beta) \sim 30$ while the mean observed ratio is about three times smaller. The predicted $H \alpha / H \beta / H \gamma$ is also in bad agreement with the observations, which is probably, a manifistation of the same problem.

Kwan and Krolik (1981) and Kwan (1984) demonstrated that a cleverly chosen ionizing continuum, characterized by a hard and intense $\mathrm{X}$-ray component, helps to alleviate the problem, at the price of predicting very weak HeII lines. Such continuua are rarely observed and those more typical of quasars fail to explain the observations.

There are clear indications that the problem is not due to the unusual weakness of $L \boldsymbol{\alpha}$ but rather the very intense Balmer lines. It is also evident that higher density models are in better agreement with the observations (e.g. Hubbard and Puetter 1985, Wills Netzer and Wills 1985, Collin-Souffrin and Dumount 1986), but these produce too weak intercombination lines. This is an obvious case where the transfer calculation may not be adequate, or else the concept of the standard model is in question.

The only alternative explanation is reddening in the AGN galaxy. The dust is probably not inside the broad line clouds, as this will result in differential depletion of resonance line photons ( $L \alpha, \mathrm{CIV} 1549$ ) relative to HeII and the intercombination lines. Dust outside the BLR presents other problems; there is no direct indication of continuum reddening in many bright quasars, while these objects show typical $L \alpha / H \beta$ ratio. There are some ways out, like special geometry, but this dust has not yet been discovered.

\subsection{The FeII Problem}

Most AGN show strong, broad optical FeII lines The integrated intensity of all $\lambda \geq 4000 A$ FeII lines is typically 2-4 times the $H \beta$ intensity, but some objects exhibit much stronger lines (e.g. Lawrence et al 1988). There have been some claims that the strength of these lines is correlated with the radio and $\mathrm{X}$-ray properties.

The overall strength of the FeII lines was not realized until the UV lines of this ion have been observed. Wills, Netzer and Wills (1985) were able to show that the UV FeII lines are extremely strong in many AGN. There are so many such lines that some theoretical or empirical modeling is needed to estimate their strength. The total Fell emission, can exceed the $L \alpha$ intensity. When convolved with a typical broad line profile they form low contrast features, that are difficult to seperate from the continuum. The most noticable FeII feature extends from 2000 to $4000 \AA$; together with the Balmer continuum and several OIII fluorescence lines, they form what is now called "The small bump" (see Fig. 1).

Not a single photoionization model can reproduce the observed FeII line intensity of a typical AGN. It is clear that the hard photons are responsible for the excitation of the lines and the $X$-ray properties may be related to this (e.g. Wilks et al 1987). AS pointed out by Joly (1987) the optical FeII lines of some objects present even a more severe challenge to AGN modelers since the discrepency with the theory can exceed a 
factor of 5. This basic failure of the standard model resulted in some interesting new alternatives that are discussed in section 5 . It is worth adding, however, that $\mathrm{Fe}^{+}$is by far the most complicated ion treated by models today. The atomic data for it is poorly known and there are complicated line fluorescence processes that are difficult to treat. Most workers in this area choose to consider only a small subset of all levels and it is not at all clear that this is adequate, especially at high densities.

\subsection{The Energy Budget Problem}

Whatever indication we have of the shape of AGN ionizing continuum, they all suggest a rather steep slope below $\lambda=912 A$ (e.g. Bechtold et al 1984, O'Brien 1987). The steepening observed in some high redshift objects is due to intervening $L \alpha$ clouds, but this could not be the case for the lower $\mathrm{z}$ objects studied with the IUE.

There are indirect ways to estimate the shape of the UV continuum. MacAlpine et al (1985) used the observed equivalent width of HeII 1640 to argue that the mean spectral index between 1 and 4 Rydberg is about 1 . This estimate is based, of course, on the validity of the standard model, especially on previous knowledge of the covering factor. Netzer (1985) noted that most model do not produce the observed total energy in all emission lines and suggested a general way to deduce the continuum slope. In optically thick situation the total emergent flux equals the ionizing flux absorbed by BLR clouds, and the L $\alpha$ intensity is (given the assumptions of the standard model) a good measure of the number of ionizing photons. Together they give a direct measure of the mean energy of an ionizing photon, i.e. the slope of the Lyman continuum. The results indicate that the intrinsic continuum extend into the UV with a spectral index of 0.5 or less, very different from the one observed. Collin-Souffrin (1986) later argued that even a hard 1-4 Ryd. continuum cannot solve the problem since the observed strength of the low excitation lines is too large. This is another aspect of the FeII problem.

There are several ways out. One is to assume some line reddening, by $E(B-V) \sim 0.25$ mag., another is to abandon the standard model in its simplest form.

\subsection{The hot phase}

A basic question for which we have no answer yet is what determines the cloud size, pressure and column density. A novel idea, that was investigated by Krolik, McKee and Tarter (1981) and several others, is confinement by a hot gas, in pressure equilibrium with the BLR clouds. The conditions for stable equilibrium were found to be around $U=10^{-2}$, provided the X-ray continuum is the one assumed by Krolik et al. Elitzur and Ferland (1985) later showed that line radiation pressure dominates the gas pressure at $U \simeq 10^{-1.5}$ providing a natural upper limit on $U$.

Unfortunately the confining mechanism cannot work for most observed continuua, as was shown by Fabian et al (1986) and Mathews and Ferland (1987), who calculared the equilibrium temperature for the hot gas, taking into account all heating and cooling processes and using a more typical continuum. The hot phase temperature is too low and BLR clouds would move through it supersonically. Another problem is the large Compton thickness of this gas. Today it is not at all clear what holds the BLR clouds 
together. Magnetic confinement has been suggested by Rees (1988) and there are other possibilities (Krolik 1988 and section 5).

\subsection{Line variability and the BLR size}

I consider this to be the most important development in AGN study since the discovery of the hydrogen line problem, ten years ago.

As noted in section 2, the standard model makes a definite prediction about the cloud location relative to the central source, provided the values of $U$ and $N$ are known. This has been used to deduce the mass of the central object with the additional assumption of virial velocities. Since the line intensity is proportional to the ionizing luminosity, the emission lines are expected to follow the continuum variability. Such incidences have indeed occured and the light curve analysis showed delay times in bad disagreement with theoretical predictions (e.g. Peterson et al 1985, Gaskell and Sparke 1986) A comprehensive review on the subject is given in Peterson (1988).

The few cases studied so far indicate that the BLR dimension is some 2-5 times smaller than required by the standard model, i.e. the incident continuum flux (the value of $U N$ ) is some 4-25 times larger than found from the spectral analysis. This discrepency is so large that, if confirmed, it will force us to make major changes in the modeling.

Some words of caution are in order. First, the cross-correlation analysis used to obtain the BLR size ought to be improved. In particular, the uneven sampling and the poor temporal resolution of even the best studied cases, prevents us from reaching a very significant result at this stage (see Gaskell and Peterson 1987, Edelson and Krolik 1988, Maoz and Netzer 1988). Geometry dependences and non-linear response of the lines must be included in future analysis. Most important, long term monitoring, with a few days time resolution, is the only way to solve this problem. Such an attempt was recently carried out at the Wise observatory, (see Maoz this volume), but none of the galaxie had varied over the 6 month monitoring. Good research takes luck, not only wisdom.

Perhaps the most intriguing line variability observations are those reported for the very luminous AGN (Zheng et al 1988, Gonhalekar 1988), indicating very small BLR. Many of those ought to be confirmed. Also there are only 2-3 epochs per object, thus the observed line variability may be a response of the BLR to earlier continuum activity.

Some counter arguments and negative results are in place. Ulrich (1988) studied the spectrum of $3 \mathrm{C273}$ and reported no line variability during a period of large continuum changes. This objects show typical broad line spectrum and there is no apriori reason to assume that its BLR is different from that of those AGN where line variability has been reported. In another case, F-9, Clavel et al (1988) reported time lag consistent with the theory. Another negative argument concerns the line profile. Some reported line variability time scale are so short that we must worry about the basic physics involved. For instance, the Eddington luminosity provides a firm lower limit on the central mass, and the virial velocity for this mass is a lower limit on the velocity. Broad line clouds a few light days from the center of a bright AGN would show extremely broad emission lines, that are not observed. 
There is no more appropriate place to mention the large international collaboration established to monitor the UV spectrum of NGC 5548 starting December 1988. It is this kind of project that is likely to give an answer to the question of the size (and mass?) of AGN.

\section{AGN Continuum and Massive Accretion Disks}

\subsection{Thin disks, thick disks}

Part of the AGN continuum may be the result of thermal emission from the surface of a massive accretion disk. This has been proposed by Shields (1978) and later by Malkan and Sargent (1982) and others who noted the big energy excess around $3000 \AA$, nowadays called "the blue bump". Theoretical arguments for the existence of accretion disks in AGN are summarized in Begelman (1985). I shall not address the theory of AGN disks, only those aspects relevant to the BLR properties and the determination of the central mass and accretion rate.

Accretion disks come in different shapes and flavors. The classical work on $\alpha$ disks, by Shakurand Sunyaev (1973), applies to geometrically thin disks and can be used to derive their shape and spectrum. The accretion rate, express in units of the Eddington luminosity $\dot{m}=L / L_{\text {edd }}$ is 0.3 or less and larger rates result in thick disks, of different structure. Several recent papers discussed the spectrum of geometrically thin disks (Bechtold et al 1986, Czerny and Elvis 1987, Laor and Netzer 1988). They all assume "bare disks" (i.e., no coronae) and no interaction of the inner part with the outer regions. The results of the calculations are rather different, due to the different aproximations used. The only thick disk calculations applicable to AGN known to me are by Madau (1988). The present calculations are very preliminary. They do not include full atmospheric solution, scattering in a corona, radiation interaction of the different parts etc. Furthermore, the viscosity mechanism is not at all understood which makes such calculations questionable. Despite all that several general comments can be made:

a. Thin disks are hotter when the accretion rate is larger, but there is a limit on the temperature set by the maximum allowed value of $\dot{m}$

b. Electron scattering-related processes, especially the so called "modified blackbody", need to be treated with care. They tend to make the atmosphere hotter, and the spectrum harder, but the effect may not be so large when the full temperature structure of the atmosphere is taken into account (Laor and Netzer 1988). In fact, it is rather difficult to make a thin disk around a quasar black-hole hot enough to explain the observed NV 1240 and OVI 1035 lines.

c. The natural outer boundery of the thin disk is its self-gravity radius, beyond which the disk may not exist, at least its structure is no longer controlled by the central source gravity. This radius is roughly $2000 M_{9}^{-2 / 9} \dot{m}^{4 / 9} \alpha^{2 / 9}$ gravitational radii, where $M_{9}=M / 10^{9} M_{0}$.

d. Simple classical disks must be rare objects. Instabilities, winds, corona and other phenomena are likely to change their structure. In particulr, scattering by a hot corona is likely to be important and the X-ray spectrum will be affected.

e. The observed spectrum is viewing-angle dependent because of the nonisotropic geom- 
etry and relativistic effects. The gas clouds around the disk, may see a different ionizing continuum which can lead to different conditions in the gas. The observer may see different line spectrum and line and continuum variability. In this resr ect it is important to note the fundamental difference between thin and thick disks. While thin disks look the hottest when viewed edge-on (e.g. Cunningham 1975), thick disks are hotter at a face-on view, when one is looking into the central funnel. This is illustrated in Fig. 2 which shows the structure and the spectrum of thick (Madau 1988) and thin (Laor and Netzer 1988) producing thesame total luminosity.

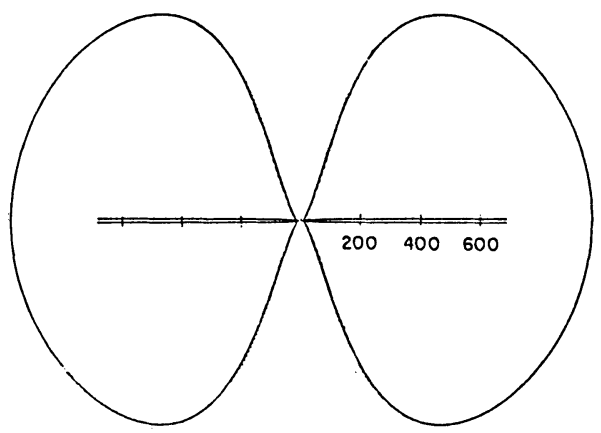

$L=7.5 \times 10^{46} \mathrm{erg} / \mathrm{sec}$
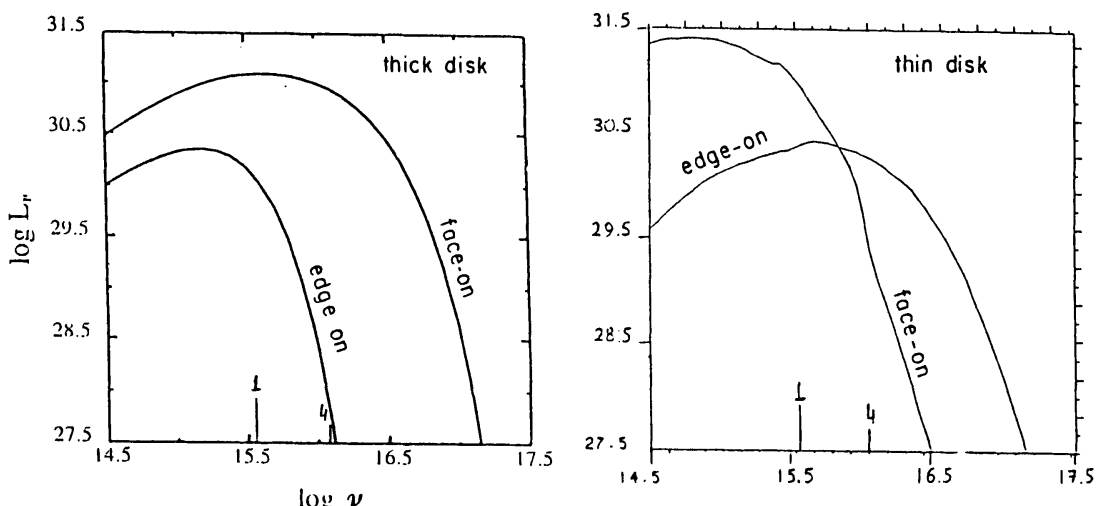

the structure (top) and spectra (bottom) of thin and thick accretion disks with total luminosity of $7.5 \times 10^{46} \mathrm{erg} / \mathrm{sec}$ (Madau 1988, Laor and Netzer 1988). Note the Lyman and $\mathrm{He}^{+}$edges marked by 1 and \& respectively.

The face-on spectrum of the two is, of course, very different, but interestingly, the face-on thick disk spectrum is not too different from the edge-on thin disk one, and vice versa. This makes it more difficult to distinguish between the two possibilities. Obviously, the emission line spectrum of the two is very different if the BLR clouds are predominantly in the plane of the disk (rotating system) or above it (a jet). 
Are there massive accretion disks in the center of AGN? The detection of the blue bump, and its detailed spectral fitting are unlikely to give a decisive answer. There is too much freedom in the theory and too short a wavelength interval to fit. Similarily, the uncertainties on $M$ and $\dot{m}$ deduced from continuum fitting are large too.

Inclination and viewing angle effects are more promising. It is possible to investigate the equivalent width distribution of a particular line, in a large sample of objects, searching for the characteristic distribution of randomly oriented disks (Netzer 1985b). (The observed luminosity of the disk is angle-dependent while that of the lines is not.) The equivalent width distribution is different for thin and thick disks, which can be used, perhaps, to distinguish between those possibilities. Related to this is the ratio of the optical and X-ray luminosity which is well-observed quantity. The thin and the thick disk models make definite and different predictions about the statistical distribution of this ratio. Polarization is another crucial test since edge-on disks are likely to be highly polarized. Realistic atmospheric solution is required.

Finally, no disk or stellar atmosphere in nature is devoid of spectral features such as absorption or emission lines and edges. Realistic calculations should predict such features. I suspect that the Lyman continuum edge will turn out to be a very useful observational tool in a search for massive disks.

\subsection{Dust and reddening}

The possible effect of dust on the observed spectrum has been mentioned in relation to the hydrogen line spectrum and the FeII problem. This section is concerned with more recent developements and I shall not address the former issue any further since it is well reviewed by MacAlpine (1985).

It is hard to imagine an astrophysical environment where cool gas is present but no dust. Simple estimates show that dust particles can survive everywhere in bright AGN except the inner parsec or so and it is natural to look for the dust signature at the infrared, where thermal emission is likely to occure. New near-IR observations of many AGN show a distinct feature near a wavelength of $3 \mu$ (e.g. Wills 1986) which has been attributed to dust emission at a temperature of about $1000 \mathrm{~K}$. Theoretical work by Barvainis (1987) show that an optically thin dust shell, located just outside the BLR, can explain this feature.

Dust emission is not the only explanation for the $3 \mu$ feature but it is a likely one,especially in AGN like F-9 by that was observed recently by Clavel, Wamsteker and Glass (1988). These authors analyzed the IR-UV continuum of F-9, over a period of several years, and found line and continuum variations, associated with a change of the $3 \mu$ continuum. Cross correlation analysis indicate that the light crossing time for the infrared continuum is about 400 days, in comparison with 160 days for the broad emission lines. A dust shell, just outside the BLR, is a likely possibility.

New polarization studies of AGN may also be relevant to the question of dust. The continuum of most radio quiet AGN is only weakly polarized, but radio-laud, flat spectrum quasars show much higher degree of polarization, presumably of nonthermal origin (e.g. Smith et al 1988). Can the residual small polarization be due to aligned dust grains? Observations in our own galaxy indicate that the degree of polarization, $p$, is 
given by $\langle p>\sim 0.05 E(B-V)$ with a very large scatter. Taken at face value the observed polarization of 1 percent may indicae a non-negligible amount of dust on our line of sight to the nucleus. Residual nonthermal polarization is a likely alternative and scattering by off-line dust or electrons, as observed in Seyfert 2s, should be considered as well.

All models involving dust must consider its effects on the UV continuum. Many quasar samples are selected such that they are heavily biased against objects with reddened continuum. It is quite likely that we are not yet aware of a large fraction of the AGN population because of this (and other) selection effects. There are already indications for reddening in faint AGN (e.g. Ward et al 1988).

\section{Recent Developements and New Ideas}

The realization of the problems listed in section 3, as well as other developments not addressed in this review, have brought about some interesting new ideas. Some are quite radical, and call for the complete replacement of the standard model, others are moderate, requiring small modifications and new viewing angles.

\subsection{Line-continuum correlations}

Correlating the line and continuum in a large sample is a powerful way to deduce properties not easily detected in individual objects. There have been a large number of such studies over the last few year and I shall mention only some results. In discussing those observations it is important to remember that the definition of the sample is very crucial. The lack of good complete samples made some authors analyse a large body of observations, randomly picked from the litrature. The advantage is the large number of objects, the disadvantage the possible selection effects. The Baldwin et al (1988) work, mentioned below, is free probably of such selection effects, all the others are not.

Several line ratios, such as CIV $1549 / L \alpha$ and CIII] $1909 /$ CIV 1549, depend on the continuum luminosity in a weak but significant way (Baldwin et al 1988, Kinney et al 1987). An interesting possibility is that the ionization parameter is luminosity dependent, as suggested by Mushotzky and Ferland (1984). If this is indeed the case than the ionization parameter in bright quasars is smaller than in faint Seyfert 1s. Unfortunately, a luminosity dependent $U$ cannot explain all the observed correlations and one must also assume a luminosity dependent covering factor (Mushotzky and Ferland 1984, Kinney et al 1987). This leads to a covering factor of almost unity for the fainter AGN, which, I suspect, will be difficult to confirm.

A different correlation which has been investigated lately is line width versus the continuum luminosity. Since $M \propto v^{2} r$ and $r$ (standard model $) \propto[N U]^{-1 / 2}$ we find $M \propto L^{1 / 2}$ if the velocity field (i.e. line width) is independent of luminosity, and $M \propto L$ if $v \propto L^{1 / 2}$ as was found by Joly et el (1985) and Joly (1987). and Wandel and Yahil (1985). These relations can be used to give $L / L_{\text {edd }}$ if the mass estimates are good.

I have great difficulties evaluating these results. The line width-continuum luminosity correlation is found for some lines $(H \alpha, H \beta)$ but not others (CIV 1549, see Joly 1987) and selections effects are strongly suspected. Furthermore, the $M-L$ calibration, used 
to deduce the value of $L / L_{\text {edd }}$ assume a known and constant value of $U N$ for all AGN and there are other simplifying assumptions. This all issue should now be rechecked in view of the new mass estimates described in section 2.

"The Baldwin Relation", relating the equivalent width of CIV 1549, and perhaps other lines, with the monochromatic continuum luminosity, is another well studied correlation (Bldwin $1977 \mathrm{~b}$, Wampler et al 1984). Currently there are three rival explanation for the observed correlation: Luminosity dependent ionization parameter and covering factor (Mushotzky and Ferland 1984, Kinney et al 1987), Continuum, but no line vriability (Murdoch 1983) and the inclination of geometrically thin disks (Netzer 1985b). There has been some confusion about these three, especially the possible selection effects involved and the reason why the relation is observed in some lines and not in others.

Finally, and most exciting, is the reltion between the radio properties and line width found by Wills and Brown (1986). It suggests that the emission line clouds in radioloud AGN move in a plane perpendiculr to the direction of the radio beam (jet). It is almost the only indication that the innermost region of AGN is intimetely connected to the larger scale structure and the relativistic motion in these objects. Further work by Browne and Murphy (1987) discusses a possible model as well as correlations of the radio and $x$-ray properties.

\subsection{Broad-Line Clouds as Bloated Stars}

The formation of the broad line clouds, and the mass supply to the central region, has been an open questions for many years. The "hot phase crisis", explained in section 3 , suggests that Comptonized gas cannot hold the clouds for sufficiently long time. This has been the motivation to suggest stellar envelopes and stellar as the BLR clouds.

Although such ideas are not entirely new, they have been revived over the last couple of years, with the work of Kazanas (1988), Penston (1988) and Scoville and Collin (1988). The range of densities predicted cover several orders of magnitude and fits naturaly the observed spectrum. The models are quite specific so there should not be any real difficulty to calculate the line spectrum. The obvious difficulty is that a large number of red giant stars is required to to explain the large covering factor. The huge number of main sequence stars expected to accompany the red giants makes the mass of the central cluster comparable to that of the central black hole. Stellar evolution in such environment is not yet understood and there are other difficulties.

Related, although different ideas have also been proposed. Terlevich and Melnick (1986) studied the evolution and spectrum of supermassive stars in a young cluster ("warmers") as the origin of activity in faint AGN. Lately they proposed that supernova explosions may provide an explanation for the line and the continuum variation in NGC 5548 and perhaps other bright Seyferts. Perry and Dyson (1987) and CollinSouffrin et al (1988) suggest that the BLR clouds are shocked gas driven by radiative acceleration and collide with some "obstacles" (stars) in the vicinity of the central black hole. No confining medium is needed in this case. It should not be difficult to put this model to a test once the properties of the shocks (clouds) are fully specified. 


\subsection{Disk emission lines}

Line emission from the central accretion disk, in analogy to the spectra of cataclysmic variables, have been proposed by Collin-Souffrin et al (1988). These authors investigated the physical conditions in the cool outer part of the central disk and suggest it could be the location of the strong FeII and other low excitation lines. Such regions could have temperatures between 2000 and $6000 \mathrm{~K}$ and are likely to be optically thin. The latest version of this model involves the heating of the region by hard $\mathrm{X}$-ray photons back scattered from the the shock fronts mentioned previously. This can raise the temperature of the disk by a few thousand degrees which, they claim, would give enough flux in the lines.

The model, as it stands today, is very different from the standard model and it remains to be seen whether it explains the observed spectrum. An obvious difficulty is the location chosen for the line producing part of the disk, which is outside the self gravity radius of the disk. It is not clear what is the density and temperature structure at this location, while these are important parameters of the calculations.

Other workers have addressed "disk emission lines" but in a very different context. Netzer (1987) suggested that the cloud distribution may be weighted towards the plane of the central disk (but much further away). Such clouds "see" a weaker but harder ionizing continuum and emit, as a result, stronger low excitation lines. The fewer clouds above the disk are subjected to a stronger radiation field and most of the higher excitation lines come from them. The ionizing flux seen by the low-latitude clouds can be very different from the one deduced by an external observer. Angle dependent photoionization is one step beyond the standard model that needs further study.

Some new observations may be in agreenent with the emission line disk idea. The $H \beta$ profile of 3C390.3, (Penston and Perez 1987) and Arp 102B (Chen et al 1988) resemble theoretical disk profiles and there are several well documented cases where the variable component of some emission lines is doubled peaked (see Peterson this volume). The Wills and Browne (1986) observations dicussed earlier is another example of a flat geometry. Needless to say, not every flat configuration is a rotating disk and there are other ways to explaine a double peak emission line (e.g. Gaskell 1983.)

Perhaps the most interesting aspect of the disk related geometry is the fact that some AGN are very similar in all their properties, but they do not look alike since we happen to observe them from a different aspect angle.

\subsection{Small, dense BLR}

Most photoionization calculations concentrate on a narrow range of $\mathrm{U}$ and $\mathrm{N}$ that give the best agreement with the observations. There have been very few calculations for densities much greater than $10^{10} \mathrm{~cm}^{-3}$ and the physics needed to describe such clouds has not been fully developed. The new line-continuum reverberation measurements emphasize the need to investigate the regime of larger $U$ and $N$ in a more systematic way and to find the smallest distance where significant contribution to the broad line emissivity is still important.

Lately we (Rees, Netzer and Ferland 1988) have undertaken a project of calculating the BLR spectrum and cloud distribution at all densities up to $10^{13} \mathrm{~cm}^{-3}$. Many physical 
processes not treated before have been added, such as three body recombination for all elements and free-free heating. We specify the pressure and velocity laws and calculate the contribution to the line emission from all densities and distances. Some of these models result in a covering factor that is heavily weighted towards the outside of the BLR. Such models are not too different from the "usual" standard model. In other cases much of the line emission comes from the very high density clouds, and lines like CIII] 1909 tend to be weak. There is no way to produce a realistically looking spectrum from high density clouds only, since at such densities much of the cooling is due to high excitation lines and diffuse continua (e.g. Paschen continuum, CIII 977) that are not observed to be strong. Such "modified standard models" are still to be confronted with the new line variability observations.

\section{Conclusions}

Only few of the fundamental questions presented in the first section can be answered with some certainty:

The best estimates of the central mass is obtained when the new line reverberation measurements are used as size indicators. The few available measurements are indicative of a very small BLR but are not yet very significant. Most disturbing are some null results that are in conflict with the reported small dimensions, and the need to assume very different BLR for very similar objects. Actual mass estimates are uncertain also because of the unknown velocity field. In any case the central mass is probably smaller than deduced by the standard model.

The accretion rate is best estimated by line-continuum correlations. The scatter in such diagrams is so large that no firm conclution can be reached. Estimates based on spectral fits to a theoretical disk specrum are even less reliable. My guess is that $L / L_{\text {edd }}$ is between 0.01 and 0.1 .

How to detect accretion disks? Probably not by their continuum spectrum. Inclination related effects are more promissing.

Future study of AGN is likely to be quite different. Small and denser regions will be studied and we will have to get use to the idea that similar objects may look different to us if we happen to look at them from different angles.

I am grateful to B.Wills, B.Peterson, G.Ferland and J.Krolik for useful comments and suggestions. This work is supported by the U.S-Israel Binational Foundation grant no. $85 / 00085$. 


\section{References}

Averett,E.H. and Loeser,R.,1988, Ap.J.,331,211.

Baldwin,J.A.,1977a,MNRAS,178,67p.

Baldwin,J.A.,1977b,Ap.J.,266,1.

Baldwin,J.A.,Wampler,J.E.,nd Gaskell,C.M.,1988,Ap.J (in press)

Bechtold,J.,Green,R.F.,Weymann,R.J.,Schmidt,M., Estabrook,F.B.,Sherman,R.D., Wahlquist,H and Heckman,T., 1984,Ap.J.,281,76.

Bechtold,J.,Czerny,B.,Elvis,M.,Fabbiano,G.,and Green,R.F., 1987,Ap.J.,314,699.

Begelman,M.C.,1985, in Astrophysics Of Active Galaxies and QSOs, (J.Miller ed.), p411.

Barvainis,R.,1987,Ap.J.,320,537.

Chen,K.,Halpern,J.P.,and Filippenko,A.V.,1988 (preprint)

Clavel,J.,Wamsteker,W., and Glass,I, 1988 (preprint)

Collin-Souffrin,S.,1986,Astr.Ap.166,115.

Collin-Souffrin,S.,Dyson,J.E., McDowell,J.C, and Perry,J.J.,1988, MNRAS,232,539.

Collin-Souffrin,S.and Dumont,S.,1986,Astr.Ap.,166,13.

Czerny,B,and Elvis,M.,1987,Ap.J.,312,325.

Cunningham,C.T.,1975,Ap.J.,202,788.

Davidson,K., 1972, Ap.J.,171,213.

Davidson,K.,and Netzer,H. 1979,Rev.Mod.Phys.,51,715.

Edelson,R.A., and Krolik,J.H., 1988 (preprint)

Elitzur,M., and Ferland,G.J., 1985,Ap.J.

Fabian,A.C.,Guilbert,P.W.,Arnud,K. ,Shafer,R.A., Tennant,A.F. and Ward,M.J., 1986, MNRAS,218,457.

Ferland,G.J.,and Rees,M.,1988 Ap.J. (in press)

Ferland ,G.J. and Shields,G.A., 1985 in Astrophysics of Active Galaxies and QSOs (J.Miller ed) p.157.

Gaskel,C.M.,1983,Liege,Ast.Coll.,24,473.

Gaskell,C.M.,and Peterson,B.M.,1987,Ap.J.Supp.,65,1.

Gaskell,C.M.,and Sparke,1986,Ap.J.,305,175.

Gondhalekar,P.M.,1988, (submitted to MNRAS)

Hubbard,E.M.,nd Puetter,R.C.,1985,Ap.J.,290,394.

Joly,M.,Collin-Souffrin,S.,Masnau,J.L., and Nottale,L., 1985,Astr.Ap.152,282.

Joly,M., 1987, in Emission Lines in Active Galactic Nuclei, (P.Gondhalekar ed. p160)

Joly,M.,1987,Astr.Ap.184,33.

Kazanas,D.,1988 (preprint)

Kinney,A.L.,Huggins,P.J.,Glassgold,A.E., and Bregman,J.N., 1987,Ap.J.,314,145.

Krolik,J.H.,1988,Ap.J.325,182.

Krolik,J.,McKee,C.M.,and Tarter,C.B.,1981,Ap.J.249,422.

Kwan,J., and Krolik,J.H. 1981, Ap.J.,250,478.

Laor,A., and Netzer,H.,1988, (MNRAS, submitted)

Lawrence,A. et al,1988, MNRAS (submitted)

Madau,P.,1988,Ap.J.,327,116.

Maoz,D.,and Netzer,H.,1988, (MNRAS, accepted)

MacAlpine,G.M., 1972,Ap.J.,175,11. 
MacAlpine,G.M.,1985 in Astrophysics of Active Galaxies and QSOs, (J.Miller ed). p.259. MacAlpine,G.M.Davidson,K.,Gull,T.R.,and Wu,C.C., 1985, Ap.J.,294,147.

Malkan,M.A.,and Sargent,W.C.W.,1982,Ap.J.254,122.

Mathews,W.G,and Ferland,G.J.,1987,Ap.J.

Mathews,W.G.,and Capriotti,E.R.,1985, in strophysics of Active Galaxies and QSOs (J.Miller ed) p.185.

Mushotzky,R.F., and Ferland G,J.,1984,Ap.J.278,558.

Netzer,H. 1985a, Ap.J. 289,451.

Netzer,H.,1985b,MNRAS,216,63.

Netzer,H. 1987,MNRAS.,225,55.

O'Brien,P.T.,1987, in Emission Lines in Active Galactic Nuclei (P.Gondhalekar ed), p35.

Penston,M.V.,1988,MNRAS,233,601.

Perez,E.,Penston,M.V.,Tadhunter,C.,Mediaville,E.,and Moles,M.,1988,MNRAS,230,353.

Peterson,B.M.,1988,Pub.Astr.Soc.Pac.,100,18.

Peterson,B.M.,Meyers,K.A.,Capriotti,E.R.,Foltz,C.B., Wilkes,B.J.,and Miller,H.R.,1985,Ap.J.,2

Rees,M.,1987,MNRAS,228,47p.

Rees,M.,Netzer,H., and Ferland,G.J., (in preparation).

Scoville,N.,and Collin,A.N.,1988 (preprint)

Shakura,N.I.,and Sunyaev,R.A.,1973,Astr.Ap.,24,337.

Shields,G.A.,1978,Nature,272,706.

Smith,P.S.,Elston,R.,Berrinan,G.,and Allen,R.G.,1988, Ap.J.Lett.,326,L39.

Wampler,E.J.,Gaskell,C.M.,Burk,W.L., and Baldwin,J.A., 1984,Ap.J.,276,403.

Wandel,A.,and Yahil,A.,Ap.J.,295,L1.

Ward,M.J.,Done,C.,Fabian,A.C.,Tennant,A.F., and Shafer,R.A., 1987,Ap.J.,

Wilkes,B.J.,Elvis,M.,and McHardy,I.,1987,Ap.J.Lett.321,L23.

Wills,B.J.,1985 (Proc. of IAU symp. no. 110, p279)

Wills,B.J.,Netzer,H., and Wills,D.1985,Ap.J.,288,94.

Wills,B.J., and Browne,I.W.,1986,Ap.J.,302,56.

Zheng,W.,Burbidge,E.M.,Smith,H.E., Cohe,R.D., and Bradley,S.E., 1987,Ap.J.322,164.

\section{DISCUSSION}

GASKELL I don't think you should worry too much about a conflict with the "Standard". Model for two reasons. First, as we pointed out (Gaskell and Sparke $1986)$ the variability samples gas close in and there can be quite a bit of gas $(50-75 \%$ perhaps) further out. Second we're really only finding very small sizes for very low luminosity quasars (e.g., NGC 4151). The C III] $\lambda 1909$ density limit doesn't apply here since $C I V$ is broader and more variable than $C$ III]. 